\section{Avaliação da assistência às gestantes: o caso do município de São José do Rio Preto, São Paulo, Brasil}

\section{Health care evaluation of pregnant women: the case of the municipality of São José do Rio Preto, São Paulo, Brazil}

Maria Sílvia de Moraes 1 Fátima Grisi Kujumjian 2 Francisco Chiaravalloti Neto 3 José Carlos Cacau Lopes 4

1-5 Departamento de Epidemiologia e Saúde Coletiva. Faculdade de Medicina de São José do Rio Preto. Av. Brigadeiro Faria Lima, 5416. São José do Rio Preto, SP, Brasil. CEP: 15.090-000. Tel. (17) 210.5718

\begin{abstract}
Objectives: to identify changes of health care indicators of women who gave birth in the municipality of São José do Rio Preto, between 1997 and 2001, according to the established maximum percentage of c-sections.

Methods: women who reside and gave birth in hospitals within the municipal in 1997 and 2001 were included in the study and specifically those who were treated in hospitals that primarily serve the National Health Care System. This was a descriptive cross-sectional survey using primary and secondary data sources. The primary data were collected using a questionnaire by sampling and secondary data came from birth certificates from the "Life Birth Registration System".

Results: between 1997 and 2001, a reduction in teenage pregnancies and an improvement in the education of women were seen. The number of c-sections was reduced in the evaluated hospitals and there was an increased number of prenatal consultations. However, other indicators such as birth weight, Apgar score, gestational age and the number of women who thought normal childbirth was the most recommended showed no changes. The indicators related to breastfeeding improved.

Conclusions: an improvement in some of the assessed indicators was seen, although the quality of the services in São José do Rio Preto needs to be further discussed.
\end{abstract}

Key words Services evaluation, Prenatal care, Parturition, Women's health

\section{Resumo}

Objetivos: detectar mudanças em indicadores de qualidade da assistência às mulheres que tiveram filhos no município de São José do Rio Preto, entre 1997 e 2001, a partir da fixação de percentual máximo de cesarianas.

Métodos: a população alvo foi de mulheres residentes e que tiveram filhos nos hospitais do município em 1997 e 2001 e em especial aquelas atendidas por hospitais cuja maioria de leitos era financiada pelo Sistema Único de Saúde. Trata-se de estudo descritivo com desenho de corte transversal que utilizou dados primários e secundários. Os primários foram coletados através de inquérito domiciliar realizado por amostragem e os secundários obtidos das Declarações de Nascidos Vivos do Sistema de Nascidos Vivos.

Resultados: entre 1997 e 2001 constatou-se redução da gravidez na adolescência e aumento da escolaridade das mulheres. Observou-se que as proporções de cesárea diminuíram nos hospitais selecionados e que houve aumento no número de consultas no prénatal. Não houve alterações no peso ao nascer, Apgar, idade gestacional e nas proporções das mulheres que achavam o parto normal o mais indicado. Os indicadores relacionados com a amamentação apresentaram melhoria.

Conclusões: verificou-se a melhoria em alguns dos indicadores avaliados, embora o acolhimento nos serviços mereça ser problematizado em São José do Rio Preto.

Palavras-chave Avaliação dos serviços, Cuidado pré-natal, Parto, Saúde da mulher 


\section{Introdução}

Analisando-se as causas de óbitos relacionadas à gravidez no Brasil, observa-se que as causas obstétricas diretas são responsáveis por mais de $90 \%$ dos óbitos. ${ }^{1}$ As patologias, na sua grande maioria, são de fácil controle e evitáveis por medidas de atenção à saúde. Sabe-se, também, que as cesarianas desnecessárias existentes no país são fatores importantes para o aumento dos coeficientes de mortalidade materna. 2,3

Há por isso uma preocupação das esferas governamentais e de segmentos da sociedade em melhorar o quadro de atendimento. Uma das propostas é de reorganização do sistema de saúde por meio de ações integrais dirigidas à saúde da mulher, com assistência pré-natal oportuna às necessidades da gestante $\mathrm{e}$ que tenha interação com os serviços de assistência ao parto. Além dessa preocupação, há necessidade de diminuir as taxas de cesariana, que estão entre as maiores do mundo.2,4

Em 1998 foi lançada uma portaria do Ministério da Saúde, (MS/GM 2816) que determinou o controle sobre o pagamento do percentual máximo de cesarianas em relação ao total de partos por hospital. Segundo essa portaria, as elevadas taxas de cesáreas são fatores determinantes de morbimortalidade materna e perinatal no Brasil. Assim, seria pago às Instituições um limite máximo de $30 \%$ de cesarianas entre o total dos partos financiados pelo Sistema Único de Saúde (SUS). 4

À luz dos pressupostos e das diretrizes que sustentam a formulação e implantação do SUS, o município de São José do Rio Preto, a partir de 1998, implantou programas de assistência à gestante nos hospitais que tinham a maioria dos seus leitos financiados pelo SUS (Hospital de Base, Hospital Ielar e Santa Casa de Misericórdia). Esses hospitais adotaram programas de assistência para a gestante, parturiente e puérpera, como alojamento conjunto, analgesia para o parto normal e acompanhamento de um familiar da gestante em tempo integral.

De acordo com a disponibilidade dos registros da Direção Regional de Saúde de São José do Rio Preto (DIR XXII), as taxas de cesariana do município de São José do Rio Preto vinham oscilando em torno de $80 \%$ desde o início da década de 80 . Os valores observados permitiam supor que as elevadas taxas de cesariana no município tenderiam a se manter nos anos seguintes, podendo haver, inclusive, incremento nessas, uma vez que São José do Rio Preto é referência regional em Saúde, e no caso específico deste estudo, referência para gestações de alto risco. 1,5
Segundo informações obtidas do Sistema de Informações de Nascidos Vivos (SINASC) a taxa de cesariana para os partos realizados nos três hospitais de São José do Rio Preto com a maioria dos leitos financiados pelo SUS era de $77 \%$ em 1997,6 ano anterior ao da implantação da portaria MS/GM 2816 do Ministério da Saúde. Em 2001 a taxa diminuiu para $49 \% .7$

A pergunta que se faz é se a redução das taxas de cesariana verificadas nesses hospitais veio acompanhada por melhoria de outros indicadores de saúde, ou se foi apenas uma imposição econômica por parte do governo aos hospitais, que se adequaram ao recebimento de verba. Portanto, o trabalho objetivou detectar mudanças nos indicadores que medem a qualidade da assistência pré-natal, ao parto e pós-natal das mulheres que tiveram filhos nestes hospitais de São José do Rio Preto, entre 1997 e 2001.

\section{Métodos}

\section{Área de estudo}

O estudo foi realizado em São José do Rio Preto, localizado a $442 \mathrm{Km}$ a Noroeste de São Paulo, pólo regional e município sede da $8^{a}$ Região Administrativa do Estado de São Paulo, Brasil, e da Direção Regional de Saúde de São José do Rio Preto (DIR XXII). No ano de 2001, a população do município era de 367.248 habitantes distribuídos em 434,10 $\mathrm{km}^{2}$ de área. 8

\section{População alvo}

A população alvo da pesquisa foi de mulheres moradoras do município de São José do Rio Preto que tiveram filhos nos anos de 1997 e 2001 nos hospitais da cidade e mais especificamente as que tiveram filhos nos Hospitais de Base, Ielar e Santa Casa de Misericórdia. Essas três instituições são de caráter privativo filantrópico, têm a maioria de seus leitos financiados pelo SUS e concentram mais de $95 \%$ dos partos realizados pelo Sistema no município. Os demais hospitais, de caráter privado, tinham atendimento limitado a poucos leitos destinados ao SUS e apresentaram pequeno número de partos financiados pelo Sistema (menos do que 5\% do total).

Foram escolhidos os anos de 1997 e 2001 porque a reorganização dos serviços de atenção à saúde das mulheres nesses hospitais, conforme a portaria MS/GM 2816, iniciou-se em 1998. Portanto, fixaram-se as datas de um ano anterior à implantação das mudanças (1997) e três anos após (2001). 4 


\section{Desenho do estudo}

O estudo é descritivo com desenho de corte transversal e natureza quantitativa. Foram utilizados para análise dados primários e secundários. Os primários foram coletados através de inquérito domiciliar realizado por amostragem, e os secundários foram obtidos do SINASC e tendo caráter populacional.

Em relação aos dados secundários, foram utilizadas as informações presentes nas Declarações de Nascidos Vivos (DN) da totalidade das mulheres residentes e que tiveram filhos em 1997 e 2001 nos hospitais da cidade para cálculo das proporções de cesariana. Particularmente para as mulheres que tiveram filhos nos três hospitais selecionados foram obtidas informações relativas à escolaridade, idade, número de filhos nascidos vivos, local de residência, número de consultas no pré-natal, idade gestacional, peso da criança ao nascer e Apgar de cinco minutos. Para isso foram utilizados os bancos de dados disponibilizados pela Secretaria Municipal de Saúde e Higiene de São José do Rio Preto e pelo Grupo de Vigilância Epidemiológica da Direção Regional de Saúde de São José do Rio Preto referentes ao município de São José do Rio Preto para os anos de 19976 e 2001.7 Nos casos de DN de nascidos vivos gemelares consideraram-se apenas as informações do filho mais velho.

\section{Amostragem}

Através de duas amostras probabilísticas, uma de mulheres que tiveram filhos nos três hospitais selecionados em 1997 e outra em 2001, foi realizado um inquérito domiciliar com perguntas abertas e fechadas. As mães foram indagadas sobre planejamento da gravidez, parto mais indicado, uso de método contraceptivo, orientação sobre amamentação e satisfação no pré-natal e parto. Os tamanhos das amostras $\left(\mathrm{n}_{0}\right)$, considerando-se amostragem casual sistemática, foram calculados através da fórmula abaixo:

$$
n_{0}=\frac{z^{2} \times p \times(1-p)}{d^{2}}
$$

\section{$\mathrm{z}=1,96$}

(correspondente ao nível de confiança de 95\%);

$\mathrm{p}=0,50$

(proporção prevista);

$\mathrm{d}=0,09$

(precisão), o que resultou em um $\mathrm{n}_{0}$ igual a 119

mulheres.
Considerando-se que as populações atendidas pelos três hospitais eram, respectivamente para 1997 e 2001, de 3355 e 3283 mulheres, fez-se a correção para a população finita e obtiveram-se tamanhos de amostras próximos a 115 mulheres. Com uma estimativa de perda de $25 \%$, foram sorteadas duas amostras, cada uma com 153 mulheres. Para cada ano as DN dos três hospitais foram agrupadas por mês e por hospital e enumeradas. As seqüências dos hospitais foram obtidas por sorteio. Uma vez que as populações estavam ordenadas procedeu-se o sorteio sistemático das amostras.

\section{Análise dos dados}

Os bancos de dados com as DN foram tabulados através do programa Epi-info, obtendo-se as frequiências absolutas e relativas das variáveis de interesse. Como se tratava de dados populacionais, as frequiências foram apresentadas e comparadas diretamente sem a necessidade de aplicação de testes estatísticos.

Com as informações obtidas do inquérito domiciliar construiu-se um banco de dados que foi analisado através do programa Epi-info. Foram levantadas as frequiências absolutas e relativas das variáveis de interesse. Como se tratava de dados amostrais, foram também realizados testes de Qui-quadrado com nível de significância de 5\%.

\section{Resultados}

No total foram consideradas, respectivamente para os anos de 1997 e 2001, as informações de 5383 e 4802 mulheres residentes e que tiveram filhos nos hospitais do município. Especificamente para os três hospitais de interesse foram consideradas, respectivamente para 1997 e 2001, as informações de 3354 e 3283 mulheres. Os tamanhos das amostras obtidas foram de 73 mulheres para 1997 e 106 para 2001. Enquanto que para este ano o número se aproximou do fixado (115), em 1997 houve uma perda maior do que a esperada. O principal motivo foi a mudança de endereço.

Com vista a explorar as informações obtidas através do SINASC, apresentamos na Tabela 1 as variáveis de caracterização as mulheres que tiveram filhos nos anos de 1997 e 2001 nos três hospitais de interesse. A grande maioria das mulheres tinha idade de 20 ou mais anos, respectivamente $71,7 \%$ e $77,2 \%$ para os dois anos. Notou-se em 1997 uma maior proporção de mulheres com menos de 20 anos em comparação com 2001. Em 1997 a maioria das mulheres tinha entre um e sete anos de estudo $(59,7 \%)$ e em 
2001 a maioria passou a ter oito ou mais anos de estudo, verificando-se grande aumento em relação a 1977, no geral esta freqüência era de apenas 15,6\%. Nota-se também, em relação a essa variável a melhoria da qualidade da informação pois, em 1997, 23,4\% não informaram a escolaridade contra 0,3\% em 2001.
Entre 1997 e 2001 praticamente não houve alteração em relação ao número de filhos nascidos vivos anteriores, cerca de $45 \%$ das mulheres não tinham filhos nascidos vivos e entre 52 e $53 \%$ tinha um a três filhos.

\section{Tabela 1}

Caracterização das mulheres que tiveram filhos nos hospitais de Base, lelar e Santa Casa de Misericórdia quanto à faixa etária, anos de estudo e filhos nascidos vivos. São José do Rio Preto, São Paulo, 1997 e 2001.

\begin{tabular}{|c|c|c|c|c|}
\hline \multirow{2}{*}{ Variáveis } & \multicolumn{2}{|c|}{19976} & \multicolumn{2}{|c|}{20017} \\
\hline & $\mathrm{n}$ & $\%$ & $\mathrm{~N}$ & $\%$ \\
\hline \multicolumn{5}{|l|}{ Faixa etária } \\
\hline$<$ de 20 anos & 918 & 27,4 & 733 & 22,8 \\
\hline 20 anos ou mais & 2406 & 71,7 & 2488 & 77,2 \\
\hline Ignorado & 30 & 0,9 & 0 & 0,0 \\
\hline \multicolumn{5}{|l|}{ Anos de estudo } \\
\hline Nenhum & 42 & 1,3 & 31 & 0,9 \\
\hline 1 a 7 anos & 2002 & 59,7 & 1444 & 44,0 \\
\hline 8 ou mais & 523 & 15,6 & 1797 & 54,8 \\
\hline Não informado & 787 & 23,4 & 11 & 0,3 \\
\hline \multicolumn{5}{|l|}{ Filhos nascidos vivos } \\
\hline Nenhum & 1493 & 44,5 & 1464 & 44,5 \\
\hline 1 a 3 & 1767 & 52,7 & 1709 & 52,1 \\
\hline 4 ou mais & 94 & 2,8 & 110 & 3,4 \\
\hline
\end{tabular}

Fontes: Secretaria Estadual de Saúde. Direção Regional de Saúde de São José do Rio Preto. Grupo de Vigilância Epidemiológica. Declarações de Nascidos Vivos do Sistema de Informação de Nascidos Vivos. São José do Rio Preto, 1997. [Registros em Banco de Dados]. 6 ; Secretaria Municipal de Saúde e Higiene de São José do Rio Preto. Declarações de Nascidos Vivos do Sistema de Informação de Nascidos Vivos. São José do Rio Preto, 2001.

[Registros em Banco de Dados].7

As informações obtidas do inquérito domiciliar a respeito de planejamento familiar mostraram (Tabela 2) que em 1997 e 2001 respectivamente $41,1 \%$ e $46,2 \%$ das mulheres planejaram a gravidez ( $p=0,516)$ e que os métodos contraceptivos mais utilizados em suas vidas reprodutivas foram em primeiro lugar a pílula, 79,4\% em 1997 e 74,5\% em
2001 ( $p=0,445$ ) e em segundo lugar (a) o condom, $32,9 \%$ em 1997 e $34,0 \%$ em 2001 ( $p=0,880)$. Os demais métodos apresentaram freqüências inferiores a $10 \%$. Quando perguntadas sobre o melhor método para evitar a gravidez, a pílula foi a resposta mais freqüente, 46,6\% em 1997 e 64,2\% em 2001 $(p=0,019)$. 
Mulheres que tiveram filhos nos hospitais de Base, lelar e Santa Casa de Misericórdia segundo respostas a perguntas sobre planejamento familiar e pré-natal obtidas por inquéritos domiciliares, São Jose do Rio Preto, 1997 e 2001.

\begin{tabular}{|c|c|c|c|c|c|c|c|}
\hline \multirow{2}{*}{$\begin{array}{l}\text { Respostas das mulheres } \\
\text { entrevistadas }\end{array}$} & \multirow{2}{*}{ Ano } & \multicolumn{2}{|c|}{ Sim } & \multicolumn{2}{|c|}{ Não } & \multirow{2}{*}{ Qui-quadrado } & \multirow{2}{*}{ Valor de $p$} \\
\hline & & $\mathrm{n}$ & $\%$ & $\mathrm{n}$ & $\%$ & & \\
\hline \multirow[t]{2}{*}{ Afirmaram ter planejado da gravidez } & 1997 & 30 & 41,1 & 43 & 58,9 & 0,36 & 0,516 \\
\hline & 2001 & 49 & 46,2 & 57 & 53,8 & & \\
\hline Afirmaram ser a pílula o primeiro método & 1997 & 58 & 79,4 & 15 & 20,6 & 0,58 & 0,445 \\
\hline de contracepção mais utilizado & 2001 & 79 & 74,5 & 27 & 25,5 & & \\
\hline Afirmaram ser a camisinha o segundo & 1997 & 24 & 32,9 & 49 & 67,1 & 0,02 & 0,880 \\
\hline método de contracepção mais utilizado & 2001 & 36 & 34,0 & 70 & 66,0 & & \\
\hline Afirmaram ser a pílula o melhor método & 1997 & 34 & 46,6 & 39 & 53,4 & 5,45 & 0,019 \\
\hline contraceptivo & 2001 & 68 & 64,2 & 38 & 35,8 & & \\
\hline Iniciaram o pré-natal por volta do terceiro & 1997 & 39 & 53,4 & 34 & 46,6 & 0,04 & 0,840 \\
\hline mês de gravidez & 2001 & 55 & 51,9 & 51 & 48,1 & & \\
\hline Afirmaram estar satisfeitas com o & 1997 & 32 & 43,8 & 41 & 56,2 & 0,66 & 0,417 \\
\hline atendimento no pré-natal & 2001 & 53 & 50,0 & 53 & 50,0 & & \\
\hline
\end{tabular}

Na Tabela 3 são apresentadas informações do SINASC sobre o número de consultas realizadas no pré-natal, onde se observa aumento expressivo de mulheres com sete ou mais consultas em 2001 (73,1\%) quando comparado com 1997 (46,3\%) e quase que total eliminação, entre esses dois anos, das categorias "não informado" e "ignorado". Infor- mações obtidas do inquérito mostraram (Tabela 2) que a maioria das mulheres iniciou o pré-natal por volta do terceiro mês de gravidez, $53,4 \%$ e $51,9 \%$ respectivamente em 1997 e $2001(p=0,840)$. Das mulheres entrevistadas, $43,8 \%$ e $50,0 \%$, respectivamente para 1997 e 2001, declararam-se satisfeitas com o atendimento no pré-natal $(p=0,417)$.

Tabela 3

Mulheres que tiveram filhos nos hospitais de Base, lelar e Santa Casa de Misericórdia segundo número de consultas no pré-natal. São José do Rio Preto, São Paulo, 1997 e 2001.

\begin{tabular}{lccrc}
\hline & \multicolumn{2}{c}{19976} & 20017 \\
\cline { 2 - 5 } Número de consultas ao pré-natal & $\mathrm{n}$ & $\%$ & $\mathrm{n}$ & \\
\hline Nenhuma & 37 & 1,1 & 29 & 0,9 \\
1 - 7 consultas & 1202 & 35,8 & 853 & 26,0 \\
7 e mais consultas & 1554 & 46,3 & 0 & 0,0 \\
Não informado & 246 & 7,3 & 2 & 0,1 \\
Ignorado & 315 & 9,4 & 3283 & 100,0 \\
Total & 3354 & 100,0 & 2399 \\
\hline
\end{tabular}

Fontes: Secretaria Estadual de Saúde. Direção Regional de Saúde de São José do Rio Preto. Grupo de Vigilância

Epidemiológica. Declarações de Nascidos Vivos do Sistema de Informação de Nascidos Vivos. São José do Rio Preto, 1997.

[Registros em Banco de Dados].6 ; Secretaria Municipal de Saúde e Higiene de São José do Rio Preto. Declarações de Nascidos

Vivos do Sistema de Informação de Nascidos Vivos. São José do Rio Preto, 2001. [Registros em Banco de Dados].7 
Na Tabela 4 são apresentadas as taxas de cesariana do município de São José do Rio Preto dos anos de 1997 e 2001, obtidos a partir do SINASC. Ressalta-se que foi apenas no agregado do Hospital de Base, Hospital Ielar, Santa Casa de Misericórdia que ocorreu a diminuição das taxas de cesariana. No grupo de hospitais com pequena porcentagem de leitos financiados pelo SUS as taxas de cesariana apresentaram aumento, 94,6\% em 1997 para 96,7\% em 2001

\section{Tabela 4}

Tipo de parto de mulheres que tiveram filhos em hospitais com maioria de leitos financiados pelo Sistema Único de Saúde (Base, lelar e Santa Casa de Misericórdia) e outros hospitais, São José do Rio Preto, 1997 e 2001.

\begin{tabular}{|c|c|c|c|c|c|c|c|c|}
\hline \multirow{3}{*}{ Tipo de parto } & \multicolumn{4}{|c|}{$\begin{array}{c}\text { Hospital de Base, Hospital lelar } \\
\text { e Santa Casa de Misericórdia }\end{array}$} & \multicolumn{4}{|c|}{ Outros hospitais } \\
\hline & \multicolumn{2}{|c|}{19976} & \multicolumn{2}{|c|}{20017} & \multicolumn{2}{|c|}{19976} & \multicolumn{2}{|c|}{20017} \\
\hline & $\mathrm{n}$ & $\%$ & $\mathrm{n}$ & $\%$ & $\mathrm{n}$ & $\%$ & $\mathrm{n}$ & $\%$ \\
\hline Normal & 777 & 23,2 & 1678 & 51,1 & 107 & 5,3 & 50 & 3,3 \\
\hline Cesariana & 2569 & 76,6 & 1604 & 48,9 & 1920 & 94,6 & 1469 & 96,7 \\
\hline Não informado & 8 & 0,2 & 1 & 0,0 & 2 & 0,1 & 0 & 0,0 \\
\hline Total & 3354 & 100,0 & 3283 & 100,0 & 2029 & 100,0 & 1519 & 100,0 \\
\hline
\end{tabular}

Fontes: Secretaria Estadual de Saúde. Direção Regional de Saúde de São José do Rio Preto.

Grupo de Vigilância Epidemiológica. Declarações de Nascidos Vivos do Sistema de Informação de Nascidos Vivos.

São José do Rio Preto, 1997. [Registros em Banco de Dados]. 6 ; Secretaria Municipal de Saúde e Higiene de São José do Rio Preto. Declarações de Nascidos Vivos do Sistema de Informação de Nascidos Vivos. São José do Rio Preto, 2001.

[Registros em Banco de Dados].7

Com relação ao parto (Tabela 5 ), $56,1 \%$ das mulheres entrevistadas e que tiveram filhos em 1997 acharam o parto normal o mais indicado; para o ano de 2001 a proporção encontrada foi de $64,1 \%$ $(p=0,282)$. Para os anos de 1997 e 2001 , respectivamente $43,8 \%$ e $32,1 \%$ das mulheres afirmaram que o parto normal era muito dolorido e apresentava maiores riscos que a cesariana $(p=0,109)$. Em 1997 , $91,8 \%$ das mulheres afirmaram ter ficado satisfeitas com atenção recebida durante o parto, contra $67,9 \%$ em $2001(p<0,001)$. O principal problema citado nos dois anos foi a falta de atenção dos profissionais médicos.

Verificou-se, através do inquérito (Tabela 5), que $49,3 \%$ e $61,3 \%$ das mulheres, respectivamente para os anos de 1997 e 2001, receberam orientação no pré-natal sobre amamentação $(\mathrm{p}=0,111)$. Das mães entrevistadas que tiveram filhos em 1997, $68,5 \%$ declararam que deveriam amamentar com exclusividade o bebê no período de seis meses a um ano, em 2001 esta proporção foi de 83,0\% ( $\mathrm{p}=0,023)$. Observou-se que $52,1 \%$ e $34,9 \%$ das mulheres, respectivamente para os anos de 1997 e 2001, alegaram dificuldades em realizar as orientações recebidas tanto no pré-natal como no hospital sobre amamentação $(p=0,022)$. Os motivos mais citados foram: início do trabalho fora de casa, preocupação com o corpo e o bebê não queria mais o peito. A maioria das mães amamentou por três ou mais meses, $64,3 \%$ em 1997 e $78,3 \%$ em $2001(p=0,040)$. 
Mulheres que tiveram filhos nos hospitais de Base, lelar e Santa Casa de Misericórdia segundo respostas a perguntas sobre o parto e a amamentação obtidas por inquéritos domiciliares, São Jose do Rio Preto, 1997 e 2001.

\begin{tabular}{|c|c|c|c|c|c|c|c|}
\hline \multirow{2}{*}{$\begin{array}{l}\text { Respostas das mulheres } \\
\text { entrevistadas }\end{array}$} & \multirow{2}{*}{ Ano } & \multicolumn{2}{|c|}{ Sim } & \multicolumn{2}{|c|}{ Não } & \multirow{2}{*}{ Qui-quadrado } & \multirow{2}{*}{ Valor de $p$} \\
\hline & & $\mathrm{n}$ & $\%$ & $\mathrm{n}$ & $\%$ & & \\
\hline Afirmaram ser o parto normal & 1997 & 41 & 56,1 & 32 & 43,9 & 1,16 & 0,282 \\
\hline o mais indicado & 2001 & 68 & 64,1 & 38 & 35,9 & & \\
\hline Afirmaram ser o parto normal muito e & 1997 & 32 & 43,8 & 41 & 56,2 & 2,57 & 0,109 \\
\hline dolorido e qu apresentava maiores riscos & 2001 & 34 & 32,1 & 72 & 67,9 & & \\
\hline Afirmaram ter ficado satisfeitas & 1997 & 67 & 91,8 & 6 & 8,2 & 14,18 & 0,000 \\
\hline com a atenção recebida durante o parto & 2001 & 72 & 67,9 & 34 & 32,1 & & \\
\hline Receberam orientação sobre amamentação & 1997 & 36 & 49,3 & 37 & 50,7 & 2,53 & 0,111 \\
\hline no pré-natal & 2001 & 65 & 61,3 & 41 & 38,7 & & \\
\hline Afirmaram que deveriam amamentar com & 1997 & 50 & 68,5 & 23 & 31,5 & 5,17 & 0,023 \\
\hline exclusividade no período de 6 meses a 1 ano & 2001 & 88 & 83,0 & 18 & 17,0 & & \\
\hline Alegaram dificuldades para realizar & 1997 & 38 & 52,1 & 35 & 47,9 & 5,22 & 0,022 \\
\hline as orientações recebidas sobre amamentação & 2001 & 37 & 34,9 & 69 & 65,1 & & \\
\hline Afirmaram ter amamentado por três & 1997 & 47 & 64,3 & 26 & 35,7 & 4,21 & 0,040 \\
\hline ou mais meses & 2001 & 83 & 78,3 & 23 & 21,7 & & \\
\hline
\end{tabular}

Na Tabela 6 são mostradas as variáveis peso ao nascer, Apgar cinco minutos e idade gestacional de mulheres que tiveram filhos nos três hospitais analisados, segundo o SINASC. As proporções para essas três variáveis apresentaram pequena ou nenhuma alteração entre os anos de 1997 e 2001. Para os nascidos vivos, a proporção dos com $2500 \mathrm{~g}$ ou mais ficou em torno de $91 \%$, a categoria 8 a 10 do Apgar apresentou proporção entre 96 e $97 \%$ e a idade gestacional de 37 a 41 semanas variou entre 92 e $93 \%$. 
Tabela 6

Nascidos vivos de mulheres que tiveram filhos nos hospitais de Base, lelar e Santa Casa de Misericórdia segundo peso ao nascer, Apgar e idade gestacional. São José do Rio Preto, São Paulo, 1997 e 2001.

\begin{tabular}{|c|c|c|c|c|}
\hline \multirow{2}{*}{ Variáveis } & \multicolumn{2}{|c|}{19976} & \multicolumn{2}{|c|}{20017} \\
\hline & $\mathrm{n}$ & $\%$ & $\mathrm{n}$ & $\%$ \\
\hline \multicolumn{5}{|l|}{ Peso ao nascer } \\
\hline$\leq$ a $2499 \mathrm{~g}$ & 301 & 9,0 & 307 & 9,4 \\
\hline$>2499 g$ & 3050 & 90,9 & 2976 & 90,6 \\
\hline Ignorado & 3 & 0,1 & 0 & 0,0 \\
\hline \multicolumn{5}{|l|}{ Apgar 5 minutos } \\
\hline 0 a 3 & 19 & 0,6 & 9 & 0,3 \\
\hline 4 a 7 & 94 & 2,8 & 87 & 2,7 \\
\hline 8 a 10 & 3221 & 96,0 & 3185 & 96,9 \\
\hline Não informado & 20 & 0,6 & 2 & 0,1 \\
\hline \multicolumn{5}{|l|}{ Idade gestacional } \\
\hline Menos de 22 semanas & 4 & 0,1 & 0 & 0,0 \\
\hline $22-31$ semanas & 18 & 0,5 & 53 & 1,6 \\
\hline $32-36$ semanas & 188 & 5.6 & 166 & 5,1 \\
\hline $37-41$ semanas & 3115 & 92,9 & 3027 & 92,2 \\
\hline 42 e mais & 7 & 0,2 & 14 & 0,4 \\
\hline Não informado & 22 & 0.7 & 23 & 0.7 \\
\hline Total & 3354 & 100,0 & 3283 & 100,0 \\
\hline
\end{tabular}

Fontes: Secretaria Estadual de Saúde. Direção Regional de Saúde de São José do Rio Preto. Grupo de Vigilância Epidemiológica. Declarações de Nascidos Vivos do Sistema de Informação de Nascidos Vivos. São José do Rio Preto, 1997. [Registros em Banco de Dados].6 ; Secretaria Municipal de Saúde e Higiene de São José do Rio Preto. Declarações de Nascidos Vivos do Sistema de Informação de Nascidos Vivos. São José do Rio Preto, 2001. [Registros em Banco de Dados].7

\section{Discussão}

Com relação à caracterização das mulheres que tiveram filhos nos anos de 1997 e de 2001, notou-se uma diminuição da gravidez na adolescência, que provavelmente está associada ao aumento de escolaridade das mães em 2001, quando comparado com 1997. Esse aumento é um fato bastante positivo, pois a escolaridade é um fator decisivo para uma melhor qualidade do pré-natal. Uma pesquisa realizada no estado de São Paulo9 observou que gestantes de baixa escolaridade se enquadravam na categoria de risco para baixo peso ao nascer.

Chama a atenção que a pílula continua como o método contraceptivo mais lembrado e mais usado, seguido apenas pelo condom, e que os demais métodos foram pouco citados e utilizados. A menção de apenas dois métodos como principais, deixa evidente um planejamento familiar com recursos bastante restritos, face à grande quantidade de métodos contraceptivos existentes. $10 \mathrm{Em}$ meio a esse processo, salienta-se que não houve diferenças no planejamen- to da gravidez entre os dois anos estudados. A reestruturação da atenção pré-natal ocorrida no período não foi suficiente para que a mulher planejasse com maior efetividade sua história reprodutiva. Entre 1997 e 2001 a proporção de mulheres que realizararam sete ou mais consultas de pré-natal em São José do Rio Preto aumentou em 27,0\%, enquanto na região Sudeste o aumento foi de $8,3 \%$ para o mesmo período. ${ }^{11}$ Além disso os dados sobre o pré-natal indicam que, no município, a quase totalidade das gestantes tinham acesso a esse serviço. Entretanto, isso pode não refletir a qualidade do atendimento, pois o processo de assistência, muitas vezes, não representa ganhos para a saúde. De acordo com Silver, 12 em muitos casos, as consultas são precárias e representam apenas atos simbólicos (consumo simbólico) para as mulheres.

Entre 1997 e 2001, as taxas de cesariana apresentadas pelos hospitais cujos leitos eram em sua maioria financiados pelo SUS sofreram importante declínio. Isso mostra que as medidas de contenção adotadas pelo governo em partos financiados pelo 
SUS mostraram-se eficazes, principalmente quando se compara esse resultado com o dos hospitais com pequeno número de leitos financiados pelo SUS. Entretanto, apesar da redução, as taxas de cesarianas nesses hospitais ainda são maiores que a média no Brasil e no estado de São Paulo. 11

Não houve alterações, entre os anos estudados, nas proporções das mulheres que achavam o parto normal o mais indicado e das que consideravam esse tipo de parto mais dolorido e de maior risco que a cesariana. Além disso, diminuiu a satisfação das mulheres com o parto realizado. A portaria do Ministério da Saúde contribuiu para diminuição da distorção da cesariana, principalmente aquela a pedido da mulher. Estudo realizado no município de São José do Rio Preto no ano de 1997 mostrou que 41\% das cesarianas foram realizadas por solicitação das próprias parturientes. ${ }^{5}$ Mas, por outro lado, a redução ocorreu sem a respectiva mudança de opinião das mulheres, fato que provavelmente está associado com a qualidade do serviço prestado.

Apesar das mudanças, ainda prevalecem altas taxas de cesariana no município, principalmente naqueles hospitais com pequeno número de leitos financiados pelo SUS. Essas taxas estão associadas a um determinado tipo de atenção obstétrica que privilegia intervenções programadas, muitas vezes, fruto da precariedade do pré-natal.13 Esse modelo, mesmo com todas as reformulações, tem ainda o médico como único responsável pela condução do parto e único com autoridade pelas decisões quanto à manipulação do corpo.

Apesar da proposta do SUS, que prevê a intervenção no processo saúde-doença por meio da integralidade das ações e da atuação multidiciplinar, o sistema de saúde ainda é pautado no tratamento da doença, as ações de promoção, prevenção e cura não têm integração e a atuação dos profissionais permanece segmentada. 14

A falta de integralidade dos serviços oferecidos às mulheres, em especial no período reprodutivo, pode contribuir para o aumento da mortalidade materna e perinatal, principalmente às mulheres usuárias das unidades de saúde, que muitas vezes são de baixo poder aquisitivo e que freqüentemente têm maiores problemas de saúde, necessitando de atenção de vários serviços. ${ }^{14}$

A ausência de variação em alguns dos indicadores analisados entre 1997 e 2001, como Apgar, idade gestacional e peso ao nascer, pode ser reflexo de políticas de saúde inadequadas e que não têm garantido a incorporação da integralidade e da equidade na oferta de serviços de atenção à saúde da mulher. O peso ao nascer, por exemplo, é determina- do, entre outros fatores, pela assistência que as mulheres estão expostas durante a gestação. 15

Com relação ao aleitamento, a melhora verificada nos indicadores avaliados aponta para uma prática benéfica tanto para a mulher como para o bebê. Devese destacar o aumento da proporção de mulheres que amamentaram por três meses ou mais entre os anos estudados e que atingiu o valor de 78,3\% em 2001. Esse aumento merece destaque, pois no Brasil o desmame precoce é uma prática constante. Enquanto que $96 \%$ das mulheres brasileiras iniciam a amamentação, apenas $11 \%$ delas amamentam exclusivamente no período de quatro a seis meses. 16

Entre todas as variáveis relacionadas com a amamentação, apenas as proporções de mulheres que receberam orientação no pré-natal não apresentaram diferença significante. Nesse caso obteve-se aumento nominal das proporções, de 49,3\% em 1997 para $61,3 \%$ em 2001, e valor de $p$ próximo ao nível de significância. Provavelmente, a incongruência desse resultado está relacionada com os tamanhos das amostras obtidas menores do que os valores fixados, principalmente o de 1997, que ficou bem abaixo das 115 mulheres. Os tamanhos de amostras menores que os necessários devem ser considerados, nesse caso e nos demais achados relacionados com o inquérito domiciliar, como uma das limitações deste estudo.

Observando os motivos do desmame precoce, verificou-se no estudo que alguns deles ultrapassam a atuação do serviço de saúde (trabalho fora de casa) e outros estão relacionados com rotinas inadequadas do serviço (preocupação com o corpo e o bebê não quis mais o peito). Um estudo indica que falhas na comunicação no pré-natal são alguns dos fatores que motivam a interrupção da amamentação. 17

Mediante esses dados uma reflexão que se coloca é o acolhimento nos serviços. Talvez se consiga reduzir a insatisfação das mulheres relacionada ao pré-natal e ao parto, bem como reforçar alguns conhecimentos primordiais na gestação, levando em consideração o conhecimento das mulheres, como por exemplo, a amamentação. Como é de se supor, cada mulher faz de sua história reprodutiva uma leitura própria. Baseadas em critérios pessoais, essas leituras escolhem algumas condutas e descartam outras, portanto é fundamental que os serviços de saúde acolham a mulher, para que ela possa incluílo em sua história pessoal.

Finalizando, podemos concluir que os indicadores sugerem que o enfoque de avaliação precisa incorporar, cada vez mais, os aspectos da qualidade no processo de assistência para tornar as políticas de saúde mais eficientes. 


\section{Agradecimentos}

Agradecemos às acadêmicas Camila Nascimento Leite e Simone Pereira da Silva pela colaboração na aplicação dos questionários e a Giovana Signorini pela digitação e tabulação dos dados.

\section{Referências}

1. Laurenti R. Perfil da mortalidade materna. In: Minayo MC. Os muitos Brasis. Saúde e população na década de 80 . Rio de Janeiro: Hucitec; 1999. p. 304-19.

2. Hotimsky SW, Rattner D, Venâncio SI, Bógus CM, Miranda MM. O parto como eu vejo ... ou como eu o desejo? Expectativas de gestantes usuárias do SUS acerca do parto e da assistência obstétrica. Cad Saúde Pública 2002; 18 : 1303-11.

3. Tanaka AC. A maternidade, dilema entre nascimento e morte. São Paulo: Hucitec; 1995.

4. Rattner D. Sobre a hipótese de estabilização das taxas de cesáreas do estado de São Paulo, Brasil. Rev Saúde Pública 1996; 30: 19-31

5. Moraes MS. Cesariana no município de São José do Rio Preto. In: Scavone L, organizador. Pesquisa de gênero: entre o público e o privado. Araraquara: UNESP; 2000. p. 91-106.

6. São Paulo. Secretaria Estadual de Saúde. São José do Rio Preto. Divisão Regional de Saúde. Grupo de Vigilância Epidemiológica. Declarações de Nascidos Vivos do Sistema de Informação de Nascidos Vivos: registros de banco de dados. São José do Rio Preto: A Divisão; 1997.

7. São José do Rio Preto. Secretaria de Saúde e Higiene. Declarações de Nascidos Vivos do Sistema de Informação de Nascidos Vivos: registros de banco de dados. São José do Rio Preto: A Secretaria; 2001.

8. Ministério da Saúde. Datasus. Informações sobre população [on line] 2004. Disponível em URL: http://www.datasus.gov.br [2004 jul 1]

9. Kilsztajn S, Rossbach A, Carmo MSN, Sugahara GTL. Assistência pré-natal, baixo peso e prematuridade no estado de São Paulo. Rev Saúde Pública 2003; 37: 303-10.
10. Vieira EM, Badiane R, Fabbro AL. Características do uso de métodos anticoncepcionais no estado de São Paulo. Rev Saúde Pública 2002; 36: 263-70.

11. Ministério da Saúde. A saúde reprodutiva: gravidez, assistência pré-natal, parto e baixo peso ao nascer. In: Ministério da Saúde. Saúde Brasil 2004: uma análise da situação de saúde. Brasília (DF): O Ministério; 2004. p. 69-83.

12. Silver LD. Direito à saúde ou medicalização da mulher? Implicações para a avaliação dos serviços de saúde para mulheres. In: Giffin K, Costa SH, organizadores. Questões da saúde reprodutiva. Rio de Janeiro: FIOCRUZ; 1999. p. 299-318.

13. Barbosa GP, Giffin K, Tuesta AA, Gama AS, Chor D, Orsi E, Reis ACG. Parto cesáreo: quem o deseja? Cad Saúde Pública 2003; 19: 1611-20.

14. Santos IS, Baroni RC, Minotto I, Klumb AG. Critérios de escolha de postos de saúde para acompanhamento pré-natal em Pelotas, RS. Rev Saúde Coletiva 2000; 34: 603-9

15. Mariotoni GGB, Barros A. Peso ao nascer e mortalidade hospitalar entre nascidos vivos, 1975-1996. Rev Saúde Pública 2000; 34: 71-6.

16. Giugliani ERJ. O aleitamento materno na prática clínica. J Pediatr [Rio de Janeiro] 2000; 76: 238-52.

17. Ramos CV, Almeida JAG. Aleitamento materno: como é vivenciado por mulheres assistidas em uma unidade de saúde de referência na atenção materno-infantil em Terezina, Piauí. Rev Bras Saúde Matern Infant 2003; 3: 315-21.

Recebido em 24 de setembro de 2003

Versão final apresentada em 14 de outubro de 2004

Aprovado em 28 de outubro de 2004 